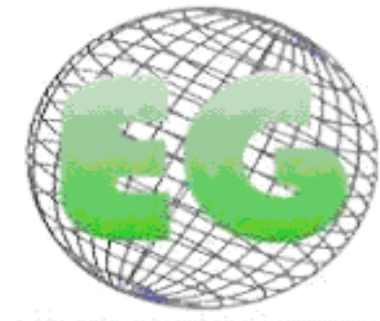

ISSN 1695-6141 $N^{\circ} 26$

www.um.es/egloball

\title{
ENSAYOS
}

\section{Modelo de gestión de casos e investigación}

Management of cases and research model

\section{"Valverde Jiménez, MR., **Hernández Corominas, MA. \\ *Enfermeras Gestoras de Casos del Servicio Murciano de Salud Área IX Vega Alta del Segura. Murcia.}

Palabras clave: atención de enfermería; gestor de salud; investigación; enfermería

Keywords: nursing care; health manager; research; nursing

\section{RESUMEN}

El modelo de gestión de casos es una forma de avanzar y mejorar la atención integrada, coordinada y continuada, centrado en la responsabilidad compartida de coordinar los cuidados, recursos, servicios y profesionales. La enfermera es líder de este proyecto por tener una visión integral orientada a cubrir necesidades y fomentar el auto-cuidado. Significa un agente de cambio en este nuevo escenario de gestión y liderazgo dentro del sistema sanitario.

El objetivo del trabajo es hacer una reflexión sobre posibles líneas de investigación en cuidados de enfermería, aplicadas a la gestión de casos. Los lenguajes NANDA-NIC-NOC constituyen un proyecto original para proporcionar un panorama general de pensamiento enfermero, una estructura y un contenido para planificar cuidados y tomar decisiones clínicas. La enfermería basada en la evidencia establece un medio útil que interconecta teoría y práctica dando instrumentos para implementar los resultados de investigación entre las enfermeras clínicas evitando la variabilidad en la práctica. El uso de la investigación enfermera enfocada a medir resultados, orientará la organización de los servicios de salud.

Las enfermeras gestoras de casos han demostrado resultados de efectividad en disminución de la mortalidad y los reingresos pero es necesario construir preguntas de investigación empleando términos y elementos del marco conceptual enfermero que aporten resultados sólidos y eviten variabilidad en la clínica.

\section{ABSTRACT}

Management of cases is a way of advancing and improving integrated, coordinates ans continuous attention, with the focus on shared responsibility in coordinating care, resourcesm services and professionals. The nurse is the leader of the project since he/she has an overall view aimed at covering needs and fostering self-care. The nurse is an agent of change in this new management and leadership scenario in the health system.

The aim of this study is to offer a refection on possible research lines on nursing cares applied to management of cases. The NANDA-NIC-NOC languages comprise an original project to provide an overview of nursing thought, a structure and content to plan care and to take clinical decisions. Evidence-based nursing establishes a useful means to interconnect theory and practice, by providing tools to implement the results from research in 
nursing clinics and so avoid variations of practice. The use of nursing research focused on measuring results can direct and guide the organization of health services.

Case manager nurses have reported results that are effective in reducing mortality and readmissions but it is necessary to develop research questions using terms and elements from the nursing conceptual framework that provide sound results and avoid variations in the clinic.

\section{INTRODUCCIÓN}

El modelo de Gestión de Casos supone una de las múltiples formas de avanzar y mejorar la Atención Integrada, Coordinada y Continuada, centrado en la responsabilidad compartida de coordinar los cuidados, recursos, servicios y profesionales ${ }^{1}$. La enfermera lidera este proyecto por tener una visión integral orientada a cubrir las necesidades, fomentar el autocuidado y con competencias en la valoración, planificación de los cuidados. Significa un agente de cambio en este nuevo escenario de gestión y liderazgo dentro del sistema sanitario ${ }^{1}$.

Las funciones que lleva a cabo son la atención y provisión de cuidados a la persona y cuidador/a, planificación, coordinación y activación de recursos, apoyo, facilitación y promoción, participación, formación, información y educación para la salud, investigación y evaluación. Según el modelo de Gestión de enfermedades de la pirámide de riesgo desarrollado por Kaiser Permanente en Estados Unidos, la Gestión de Casos engloba entre un 3 y un $5 \%$ de los pacientes de alta complejidad ${ }^{2}$

Las enfermeras gestoras de casos han demostrado resultados de efectividad en disminución de la mortalidad y los reingresos pero no se sabe si los resultados vienen determinados por un mantenimiento infectivo de la salud o vienen de un modelo de suplencia - ayuda o modelo basado en la interacción ${ }^{3}$. Es necesario construir preguntas de investigación en enfermería que empleen términos y elementos del marco conceptual enfermero y que aporten resultados sólidos ${ }^{3}$. El objetivo de este trabajo es hacer una reflexión sobre posibles líneas de investigación en cuidados de enfermería, aplicadas a la gestión de casos.

\section{GESTIÓN DE CASOS Y PROCESO ENFERMERO}

La Enfermera Gestora de Casos utiliza el proceso enfermero como método sistemático, dinámico, que tiene una base teórica para brindar cuidados humanistas, centrado en el logro de objetivos de forma eficiente. Es humanista porque mientras planificamos y ofrecemos los cuidados, debemos considerar los intereses, ideales y deseos íntimos del usuario de la familia y/o la comunidad ${ }^{4}$.

La enfermera gestora de casos utiliza un lenguaje común que es la metodología enfermera. La manera de llegar al diagnóstico enfermero adecuado es contar con colaboración del paciente y la familia. La tolerancia a la ambigüedad y la práctica reflexiva permiten que la enfermera avance en su desarrollo profesional de principiante a experta ${ }^{5}$. Una enfermera que conoce a la persona en todo su contexto biopsicosocial puede facilitar decisiones conjuntas con otros profesionales a la hora de planificar unos cuidados ${ }^{1}$, por ejemplo, el modelo Área puede servir como guía para diseñar planes de cuidados estandarizados, porque ayuda a identificar las prioridades de cuidados. El profesor Pesut ${ }^{6}$ lo define como la tercera generación en el Proceso de Atención de Enfermería, en cuanto a que pasa de un enfoque basado en problemas a un enfoque basado en resultados. 


\section{ESTANDARIZACIÓN EN EL LENGUAJE ENFERMERO}

Dentro de las prioridades de investigación en cuidados en España ${ }^{7}$ están evaluar la eficacia de las intervenciones de enfermería, la promoción de la salud: desarrollo de estrategias para implicar al usuario y cuidados basados en la evidencia: implantación y evaluación de resultados en la práctica clínica.

La Enfermería Basada en la evidencia constituye un medio útil que interconecta teoría y práctica dando instrumentos de evaluación, difusión e implementación de resultados de investigación entre las enfermeras clínicas evitando la variabilidad en la práctica. Aporta a los lenguajes estandarizados el pensamiento pragmático, breve, analítico, sencillo, operativo y resolutivo ${ }^{8}$. Kautz y Van Horn ${ }^{9}$ han ilustrado cómo los lenguajes NANDA-NIC-NOC (NNN), en pacientes con enfermedad cardiaca, pueden ser usados en el desarrollo de guías de práctica clínica basada en la evidencia, tanto para la práctica clínica como para la investigación. Según Elizabeth Swanson la NNN constituye el proyecto original para proporcionar un panorama general del pensamiento enfermero, así como la estructura y el contenido para el desarrollo del conocimiento enfermero, la planificación de cuidados, y la toma de decisiones clínicas. Esta relación proporciona un "mapa de ruta conceptual" específico para la disciplina o un proyecto importante para relacionar los diagnósticos, las intervenciones y los resultados que prepara a las enfermeras para el panorama general en la era conceptual emergente.

\section{EL NOC Y EL CUIDADO EN ENFERMERÍA}

EI NOC ${ }^{10}$ se presenta como la oportunidad de investigar en resultados en salud, desde la propia disciplina enfermera, y de medir resultados sensibles a la práctica enfermera, esto es, "el hacer por hacer, al hacer para obtener"11. Es el cambio de una práctica cuidadora sin vínculo entre la detección de problemas y el establecimiento de criterios de resultado hacia un ejercicio clínico centrado en la persona y su familia, con la búsqueda permanente de resultados derivados de la planificación conjunta de los cuidados ${ }^{11}$.

En las VIII Jornadas de trabajo AENTDE, Elisabeth Swanson concretó que la inclusión del paciente y/o familia en el proceso de toma de decisiones para la selección de los resultados del paciente, asegurará que sus preferencias sean consideradas. Tradicionalmente las decisiones en relación al paciente-familia se han tomado bajo el criterio profesional ${ }^{12}$ y se ha comprobado que esto no siempre es así y que, resultados considerados por los profesionales como muy buenos, no eran experimentados por los usuarios en la misma medida $^{13}$. Además de colaborar en la selección de los resultados, también deben colaborar en la selección de la puntuación de los indicadores así como en la determinación del nivel de cambio que el paciente quiere conseguir. Nos cuenta que la parte fundamental que guía el trabajo de la NOC es que la estandarización de la etiqueta de resultado y sus indicadores es crucial para la totalidad del proceso. La puntuación de la etiqueta del resultado proporciona a las enfermeras la evidencia que permite mostrar el efecto de las intervenciones enfermeras escogidas para tratar al paciente. Además de estudiar las relaciones entre intervenciones y resultados, las relaciones entre el entorno, la estructura de la organización del sistema sanitario, el proceso de cuidados y los resultados del paciente también deben ser estudiados. Sin este tipo de datos, las organizaciones disponen de poca información sobre la que ajustar el tipo de personal que necesitan, o determinar el coste efectividad de cambios estructurales o de procesos en la prestación de cuidados de enfermería ${ }^{14}$ 


\section{CONCLUSIONES}

Las cuestiones relacionadas con el estudio de los factores organizacionales que influyen en los resultados del paciente han ganado preponderancia en estos últimos tiempos ${ }^{14}$. La gestión de casos está situada en una posición de dar prioridad a la evaluación de la efectividad de los cuidados a partir de la utilización de términos y elementos del marco conceptual enfermero que aporten resultados sólidos a la hora de cuidar, evitando la variabilidad en la práctica clínica y así adquirir una práctica basada en la elaboración de juicios. El uso de la investigación enfermera, debe trascender de la teoría a la práctica con la aplicación de resultados y con orientación de servicios de salud conforme a los resultados de investigación. La Enfermera Gestora de Casos centrada en una línea de investigación en resultados, empleando términos y elementos del marco conceptual enfermero, podrá demostrar costo-beneficio y costo-efectividad en la prestación de servicios de salud ${ }^{15}$

\section{BIBLIOGRAFÍA}

(1)Dirección General de Asistencia Sanitaria; Enfermera Gestora de Casos en el Servicio Murciano de Salud, Programa Pilotaje 2010. disponible en: https://correo.carm.es/exchweb/bin/redir.asp?URL=http://www.ffis.es/ups/taller_gestion_caso s_2010/triptic_formacion_harvard_2009_4.pdf [ Consultado 11 febrero de 2011]

(2) Ollero M. Modelos de organización para la atención a pacientes crónicos complejos. Foro de debate. Gestión clínica 2.0. Escuela Andaluza de Salud Pública. [Internet] 2009 [consultado el 17 agosto de 2011]; disponible en: http://si.easp.es/gestionclinica/wpcontent/uploads/2009/10/Doc-2.-Modelos-de-organizacion-para-la-atencion-a-pacientescronicos-complejos-_Ollero-Baturone-M._pdf.

(3) Morales Asencio JM. ¿Qué aporta el concepto de evidencia científica a la práctica clínica de los cuidados? Index de Enfermería [Index Enferm] (edición digital) 2003; 40-41.

Disponible en <http://www.index-f.com/index-enfermeria/40-41revista/40-41_articulo_3540.php [Consultado el 18 de Junio de 2011]

(4) Rosalinda Alfaro-LeFevre, Aplicación del Proceso Enfermero. Guía paso a paso. 4a ed. Springer4. 1999

(5) Lunney, M; Valoración, juicio clínico y diagnósticos enfermeros: cómo determinar los diagnósticos adecuados. NANDA Internacional. Diagnósticos enfermeros definiciones y clasificación 2009-2011. Barcelona: Elsevier; 2010.

(6) Espinosa i Fresnedo C. Entrevista a Daniel Pesut. El modelo AREA. El modelo AREA es la evolución del PAE que se centra en los resultados. AENTDE. Disponible en: http:/www.aentde.com/modules.php?name=News\&sid=21. [Consultado el 22 mayo de 2011] (7) Comet-Cortés P, Escobar-Aguilar, G, González Gil, T, de Ormijana Saenz Hernández A, Rich Ruiz M, Vidal Thomas C, Córcoles Jiménez, P et cols Establecimiento de prioridades de investigación en enfermería en España: Estudio Delphi, Enferm Clin. 2010; 20(2):88-96

(8) Redacción Evidentia. IV Reunión sobre Enfermería Basada en la Evidencia. NANDANIC-NOC "De la perplejidad a la generación de conocimiento". Consenso de congresos. Evidentia. 2007 mar-abr; 4(14). En: http://www.index-f.com/evidentia/n14/341articulo.php [Consultado el 1 de febrero de 2011]

(9) Kautz, D.D\& Van Horn, E An exemplar of the use or NNN language in developing evidence-based guidelines. International Journal of Nursing Terminologies and Classifications, 2008 19(1), 14-19

(10) Moorhead, S; Johnson M; Maas ML; Swanson E. Clasificación de Resultados de enfermería (NOC). 4ㄹaed. Barcelona: Elsevier; 2009.

(11) Morales Asencio JM. Investigación de Resultados en Enfermería el camino hacia la efectividad de los cuidados. Index de Enfermería [Index Enferm] (edición digital) 2004; 44-45. 
Disponible en <http://www.index-f.com/index-enfermeria/44revista/44_articulo_37-41.php [Consultado el 21 de marzo de 2011]

(12) Ruland CM. Decision support for patient preference-based care-planning: effects on nursing care and patient outcomes. J Am Med Informatics Assoc, 1999; 6(4): 304-312

(13) Fowler FJ, Cleary PD, Magaziner J, Patrick DL, Benjamin K. Methodological issues in measuring patient reported outcomes: the agenda of the work group on outcome assessment. Med Care, 1994; 32(7):65-76

(14) Swanson, E; EI NOC en el proceso de cuidados. VIII Jornadas de trabajo AENTDE. Mayo de 2011

(15) Parrado Lozano, Yaneth Mercedes, investigación, columna para la práctica de enfermería. Avances en enfermería., 2010; 28(1): 11-12 